\title{
Semiregular and strongly irregular boundary points for $p$-harmonic functions on unbounded sets in metric spaces
}

\author{
Anders Björn ${ }^{1}$ (D) . Daniel Hansevi ${ }^{1}$ (D)
}

Received: 16 July 2020 / Accepted: 15 February 2021 / Published online: 28 July 2021

(c) The Author(s) 2021

\begin{abstract}
The trichotomy between regular, semiregular, and strongly irregular boundary points for $p$-harmonic functions is obtained for unbounded open sets in complete metric spaces with a doubling measure supporting a $p$-Poincaré inequality, $1<p<\infty$. We show that these are local properties. We also deduce several characterizations of semiregular points and strongly irregular points. In particular, semiregular points are characterized by means of capacity, $p$-harmonic measures, removability, and semibarriers.
\end{abstract}

Keywords Boundary regularity $\cdot$ Metric space $\cdot p$-Harmonic function $\cdot$ Semibarrier Semiregular boundary point $\cdot$ Strongly irregular boundary point

Mathematics Subject Classification Primary 31E05 - Secondary 30L99 · 35J66 · 35J92 . 49Q20

\section{Introduction}

Let $\Omega \subset \mathbb{R}^{n}$ be a nonempty bounded open set and let $f \in C(\partial \Omega)$. The Perron method provides us with a unique function $P f$ that is harmonic in $\Omega$ and takes the boundary values $f$ in a weak sense, i.e., $P f$ is a solution of the Dirichlet problem for the Laplace equation $\Delta u=0$. It was introduced on $\mathbb{R}^{2}$ in 1923 by Perron [25] and independently by Remak [26]. A point $x_{0} \in \partial \Omega$ is regular if $\lim _{\Omega \ni y \rightarrow x_{0}} \operatorname{Pf}(y)=f\left(x_{0}\right)$ for every $f \in C(\partial \Omega)$. Wiener [28] characterized regular boundary points by means of the Wiener criterion in 1924. In the same year Lebesgue [22] gave a different characterization using barriers.

This definition of boundary regularity can be paraphrased in the following way: The point $x_{0} \in \partial \Omega$ is regular if the following two conditions hold:

(i) For all $f \in C(\partial \Omega)$ the $\operatorname{limit}_{\lim _{\Omega \ni y \rightarrow x_{0}}} P f(y)$ exists.

(ii) For all $f \in C(\partial \Omega)$ there is a sequence $\Omega \ni y_{j} \rightarrow x_{0}$ such that $\lim _{j \rightarrow \infty} \operatorname{Pf}\left(y_{j}\right)=f\left(x_{0}\right)$.

Daniel Hansevi

daniel.hansevi@liu.se

Anders Björn

anders.bjorn@liu.se

1 Department of Mathematics, Linköping University, 58183 Linköping, Sweden 
Perhaps surprisingly, it is the case that for irregular boundary points exactly one of these two properties fails; one might have guessed that both can fail at the same time but this can in fact never happen. A boundary point $x_{0} \in \partial \Omega$ is semiregular if the first condition holds but not the second; and strongly irregular if the second condition holds but not the first.

For the Laplace equation it is well known that all boundary points are either regular, semiregular, or strongly irregular, and this trichotomy (in an abstract linear setting) was developed in detail in Lukeš-Malý [23]. Key examples of semiregular and strongly irregular points are Zaremba's punctured ball and the Lebesgue spine, respectively, see Examples 13.3 and 13.4 in [6].

A nonlinear analogue is to consider the Dirichlet problem for $p$-harmonic functions, which are solutions of the $p$-Laplace equation $\Delta_{p} u:=\operatorname{div}\left(|\nabla u|^{p-2} \nabla u\right)=0,1<p<\infty$. This leads to a nonlinear potential theory that has been studied since the 1960s. Initially, it was developed for $\mathbb{R}^{n}$, but it has also been extended to weighted $\mathbb{R}^{n}$, Riemannian manifolds, and other settings. In more recent years, it has been generalized to metric spaces, see, e.g., the monograph Björn-Björn [6] and the references therein. The Perron method was extended to such metric spaces by Björn-Björn-Shanmugalingam [9] for bounded open sets and Hansevi [16] for unbounded open sets.

Boundary regularity for $p$-harmonic functions on metric spaces was first studied by Björn [13] and Björn-MacManus-Shanmugalingam [14], and a rather extensive study was undertaken by Björn-Björn [5] on bounded open sets. Recently this theory was generalized to unbounded open sets by Björn-Hansevi [11]; see also Björn-Björn-Li [7]. For further references and a historical discussion on regularity for $p$-harmonic functions we refer the interested reader to the introduction in [11].

For $p$-harmonic functions on $\mathbb{R}^{n}$ and metric spaces the trichotomy was obtained by Björn [4] for bounded open sets. It was also obtained for unbounded sets in certain Ahlfors regular metric spaces by Björn-Björn-Li [7]. Adamowicz-Björn-Björn [1] obtained the trichotomy for $p(\cdot)$-harmonic functions on bounded open sets in $\mathbb{R}^{n}$.

In this paper we obtain the trichotomy in the following form, where regularity is defined using upper Perron solutions (Definition 5.1). (We use upper Perron solutions as it is not known whether continuous functions are resolutive with respect to unbounded $p$-hyperbolic sets.)

Theorem 1.1 (Trichotomy) Assume that $X$ is a complete metric space equipped with a doubling measure supporting a p-Poincaré inequality, $1<p<\infty$. Let $\Omega \subset X$ be a nonempty (possibly unbounded) open set with the capacity $C_{p}(X \backslash \Omega)>0$. If $\Omega$ is unbounded, then the point at infinity, $\infty$, is considered to belong to the boundary $\partial \Omega$ in the one-point compactification $X^{*}:=X \cup\{\infty\}$.

Let $x_{0} \in \partial \Omega \backslash\{\infty\}$. Then $x_{0}$ is either regular, semiregular, or strongly irregular for functions that are p-harmonic in $\Omega$. Moreover,

- $x_{0}$ is strongly irregular if and only if $x_{0} \in \bar{R} \backslash R$, where

$$
R:=\{x \in \partial \Omega \backslash\{\infty\}: x \text { is regular }\}
$$

and $\bar{R}$ is the closure of $R$. 
- The relatively open set

$$
S:=\left\{x \in \partial \Omega \backslash\{\infty\} \text { : there is } r>0 \text { such that } C_{p}(B(x, r) \cap \partial \Omega)=0\right\}
$$

consists exactly of all semiregular boundary points of $\partial \Omega \backslash\{\infty\}$.

The importance of the distinction between semiregular and strongly irregular boundary points is perhaps best illustrated by the equivalent characterizations given in Theorems 6.4 and 6.5. Semiregular points are in some ways not seen by Perron solutions.

Our contribution here is to extend the results in [4] to unbounded open sets. In order to do so there are extra complications, most notably the fact that it is not known whether continuous functions are resolutive with respect to unbounded $p$-hyperbolic sets. We will also rely on the recent results by Björn-Hansevi [11] on regularity for $p$-harmonic functions on unbounded sets in metric spaces. Most of our results are new also on unweighted $\mathbb{R}^{n}$.

\section{Notation and preliminaries}

We assume that $(X, d, \mu)$ is a metric measure space (which we simply refer to as $X$ ) equipped with a metric $d$ and a positive complete Borel measure $\mu$ such that $0<\mu(B)<\infty$ for every ball $B \subset X$. It follows that $X$ is second countable. For balls $B\left(x_{0}, r\right):=\left\{x \in X: d\left(x, x_{0}\right)<r\right\}$ and $\lambda>0$, we let $\lambda B=\lambda B\left(x_{0}, r\right):=B\left(x_{0}, \lambda r\right)$. The $\sigma$-algebra on which $\mu$ is defined is the completion of the Borel $\sigma$-algebra. We also assume that $1<p<\infty$. Later we will impose further requirements on the space and on the measure. We will keep the discussion short, see the monographs Björn-Björn [6] and Heinonen-Koskela-Shanmugalingam-Tyson [18] for proofs, further discussion, and references on the topics in this section.

The measure $\mu$ is doubling if there exists a constant $C \geq 1$ such that

$$
0<\mu(2 B) \leq C \mu(B)<\infty
$$

for every ball $B \subset X$. A metric space is proper if all bounded closed subsets are compact, and this is in particular true if the metric space is complete and the measure is doubling.

We say that a property holds for p-almost every curve if it fails only for a curve family $\Gamma$ with zero $p$-modulus, i.e., there exists a nonnegative $\rho \in L^{p}(X)$ such that $\int_{\gamma} \rho d s=\infty$ for every curve $\gamma \in \Gamma$. For us, a curve in $X$ is a rectifiable nonconstant continuous mapping from a compact interval into $X$, and it can thus be parametrized by its arc length $d s$.

Following Koskela-MacManus [21] we make the following definition, see also Heinonen-Koskela [17].

Definition 2.1 A measurable function $g: X \rightarrow[0, \infty]$ is a p-weak upper gradient of the function $u: X \rightarrow \overline{\mathbb{R}}:=[-\infty, \infty]$ if

$$
\left|u(\gamma(0))-u\left(\gamma\left(l_{\gamma}\right)\right)\right| \leq \int_{\gamma} g d s
$$

for $p$-almost every curve $\gamma:\left[0, l_{\gamma}\right] \rightarrow X$, where we use the convention that the left-hand side is $\infty$ whenever at least one of the terms on the left-hand side is infinite. 
One way of controlling functions by their $p$-weak upper gradients is to require a Poincaré inequality to hold.

Definition 2.2 We say that $X$ supports a p-Poincaré inequality if there exist constants, $C>0$ and $\lambda \geq 1$ (the dilation constant), such that for all balls $B \subset X$, all integrable functions $u$ on $X$, and all $p$-weak upper gradients $g$ of $u$,

$$
\frac{1}{\mu(B)} \int_{B}\left|u-u_{B}\right| d \mu \leq C \operatorname{diam}(B)\left(\frac{1}{\mu(\lambda B)} \int_{\lambda B} g^{p} d \mu\right)^{1 / p},
$$

where $u_{B}:=\frac{1}{\mu(B)} \int_{B} u d \mu$.

Shanmugalingam [27] used $p$-weak upper gradients to define so-called Newtonian spaces.

Definition 2.3 The Newtonian space on $X$, denoted $N^{1, p}(X)$, is the space of all extended real-valued functions $u \in L^{p}(X)$ such that

$$
\|u\|_{N^{1, p}(X)}:=\left(\int_{X}|u|^{p} d \mu+\inf _{g} \int_{X} g^{p} d \mu\right)^{1 / p}<\infty,
$$

where the infimum is taken over all $p$-weak upper gradients $g$ of $u$.

The quotient space $N^{1, p}(X) / \sim$, where $u \sim v$ if and only if $\|u-v\|_{N^{1, p}(X)}=0$, is a Banach space, see Shanmugalingam [27].

Definition 2.4 The Dirichlet space on $X$, denoted $D^{p}(X)$, is the space of all measurable extended real-valued functions on $X$ that have a $p$-weak upper gradient in $L^{p}(X)$.

In this paper we assume that functions in $N^{1, p}(X)$ and $D^{p}(X)$ are defined everywhere (with values in $\overline{\mathbb{R}}$ ), not just up to an equivalence class. This is important, in particular for the definition of $p$-weak upper gradients to make sense.

A measurable set $A \subset X$ can itself be considered to be a metric space (with the restriction of $d$ and $\mu$ to $A$ ) with the Newtonian space $N^{1, p}(A)$ and the Dirichlet space $D^{p}(A)$ given by Definitions 2.3 and 2.4, respectively. If $X$ is proper and $\Omega \subset X$ is open, then $u \in N_{\text {loc }}^{1, p}(\Omega)$ if and only if $u \in N^{1, p}(V)$ for every open $V$ such that $\bar{V}$ is a compact subset of $\Omega$, and similarly for $D_{\text {loc }}^{p}(\Omega)$. In general, the local spaces $N_{\text {loc }}^{1, p}(\Omega)$ and $D_{\text {loc }}^{p}(\Omega)$ are not equal. However, under the assumptions given at the beginning of Sect. 3 , it is true that $N_{\mathrm{loc}}^{1, p}(\Omega)=D_{\mathrm{loc}}^{p}(\Omega)$, by Proposition 4.14 in [6].

If $u \in D_{\mathrm{loc}}^{p}(X)$, then there exists a minimal p-weak upper gradient $g_{u} \in L_{\mathrm{loc}}^{p}(X)$ of $u$ such that $g_{u} \leq g$ a.e. for all $p$-weak upper gradients $g \in L_{\mathrm{loc}}^{p}(X)$ of $u$.

Definition 2.5 The (Sobolev) capacity of a set $E \subset X$ is the number

$$
C_{p}(E):=\inf _{u}\|u\|_{N^{1, p}(X)}^{p},
$$

where the infimum is taken over all $u \in N^{1, p}(X)$ such that $u \geq 1$ on $E$.

A property that holds for all points except for those in a set of capacity zero is said to hold quasieverywhere (q.e.). 
The capacity is countably subadditive, and it is the correct gauge for distinguishing between two Newtonian functions: If $u \in N^{1, p}(X)$, then $u \sim v$ if and only if $u=v$ q.e. Moreover, if $u, v \in N_{\text {loc }}^{1, p}(X)$ and $u=v$ a.e., then $u=v$ q.e.

Continuous functions will be assumed to be real-valued unless otherwise stated, whereas semicontinuous functions are allowed to take values in $\overline{\mathbb{R}}$. We use the common notation $u_{+}=\max \{u, 0\}$, let $\chi_{E}$ denote the characteristic function of the set $E$, and consider all neighbourhoods to be open.

\section{The obstacle problem and $p$-harmonic functions}

We assume from now on that $1<p<\infty$, that $X$ is a complete metric measure space supporting a p-Poincaré inequality, that $\mu$ is doubling, and that $\Omega \subset X$ is a nonempty (possibly unbounded ) open subset with $C_{p}(X \backslash \Omega)>0$.

Definition 3.1 A function $u \in N_{\text {loc }}^{1, p}(\Omega)$ is a minimizer in $\Omega$ if

$$
\int_{\varphi \neq 0} g_{u}^{p} d \mu \leq \int_{\varphi \neq 0} g_{u+\varphi}^{p} d \mu \quad \text { for all } \varphi \in N_{0}^{1, p}(\Omega),
$$

where $N_{0}^{1, p}(\Omega)=\left\{\left.u\right|_{\Omega}: u \in N^{1, p}(X)\right.$ and $u=0$ in $\left.X \backslash \Omega\right\}$. Moreover, a function is $p$-harmonic if it is a continuous minimizer.

Kinnunen-Shanmugalingam [20, Proposition 3.3 and Theorem 5.2] used De Giorgi's method to show that every minimizer $u$ has a Hölder continuous representative $\tilde{u}$ such that $\tilde{u}=u$ q.e. Björn-Marola [12, p. 362] obtained the same conclusions using Moser iterations. See alternatively Theorems 8.13 and 8.14 in [6].

The following obstacle problem is an important tool. In this generality, it was considered by Hansevi [15].

Definition 3.2 Let $V \subset X$ be a nonempty open subset with $C_{p}(X \backslash V)>0$. For $\psi: V \rightarrow \overline{\mathbb{R}}$ and $f \in D^{p}(V)$, let

$$
\mathscr{K}_{\psi, f}(V)=\left\{v \in D^{p}(V): v-f \in D_{0}^{p}(V) \text { and } v \geq \psi \text { q.e. in } V\right\},
$$

where $D_{0}^{p}(V)=\left\{\left.u\right|_{V}: u \in D^{p}(X)\right.$ and $u=0$ in $\left.X \backslash V\right\}$. We say that $u \in \mathscr{K}_{\psi, f}(V)$ is a solution of the $\mathscr{K}_{\psi, f}(V)$-obstacle problem (with obstacle $\psi$ and boundary values $f$ ) if

$$
\int_{V} g_{u}^{p} d \mu \leq \int_{V} g_{v}^{p} d \mu \quad \text { for all } v \in \mathscr{K}_{\psi, f}(V) .
$$

When $V=\Omega$, we usually denote $\mathscr{K}_{\psi, f}(\Omega)$ by $\mathscr{K}_{\psi, f}$.

The $\mathscr{K}_{\psi, f}$-obstacle problem has a unique (up to sets of capacity zero) solution whenever $\mathscr{K}_{\psi, f} \neq \varnothing$, see Hansevi [15, Theorem 3.4]. Furthermore, there is a unique lsc-regularized solution of the $\mathscr{K}_{\psi, f}$-obstacle problem, by Theorem 4.1 in [15]. A function $u$ is lsc-regularized if $u=u^{*}$, where the lsc-regularization $u^{*}$ of $u$ in $\Omega$ is defined by 


$$
u^{*}(x)=\text { ess } \lim _{y \rightarrow x} \inf u(y):=\lim _{r \rightarrow 0} \underset{B(x, r)}{\operatorname{ess} \inf } u, \quad x \in \Omega .
$$

If $\psi: \Omega \rightarrow[-\infty, \infty)$ is continuous as an extended real-valued function, and $\mathscr{K}_{\psi, f} \neq \varnothing$, then the lsc-regularized solution of the $\mathscr{K}_{\psi, f}$-obstacle problem is continuous, by Theorem 4.4 in [15]. Hence the following generalization of Definition 3.3 in Björn-Björn-Shanmugalingam [8] (and Definition 8.31 in [6]) to Dirichlet functions and to unbounded sets makes sense. It was first used by Hansevi [15, Definition 4.6].

Definition 3.3 Let $V \subset X$ be a nonempty open set with $C_{p}(X \backslash V)>0$. The p-harmonic extension $H_{V} f$ of $f \in D^{p}(V)$ to $V$ is the continuous solution of the $\mathscr{K}_{-\infty, f}(V)$-obstacle problem. When $V=\Omega$, we usually write $H f$ instead of $H_{\Omega} f$.

Definition 3.4 A function $u: \Omega \rightarrow(-\infty, \infty]$ is superharmonic in $\Omega$ if

(i) $u$ is lower semicontinuous;

(ii) $u$ is not identically $\infty$ in any component of $\Omega$;

(iii) for every nonempty open set $V$ such that $\bar{V}$ is a compact subset of $\Omega$ and all $v \in \operatorname{Lip}(\bar{V})$, we have $H_{V} v \leq u$ in $V$ whenever $v \leq u$ on $\partial V$.

A function $u: \Omega \rightarrow[-\infty, \infty)$ is subharmonic if $-u$ is superharmonic.

There are several other equivalent definitions of superharmonic functions, see, e.g., Theorem 6.1 in Björn [2] (or Theorem 9.24 and Propositions 9.25 and 9.26 in [6]).

An lsc-regularized solution of the obstacle problem is always superharmonic, by Proposition 3.9 in [15] together with Proposition 7.4 in Kinnunen-Martio [19] (or Proposition 9.4 in [6]). On the other hand, superharmonic functions are always lsc-regularized, by Theorem 7.14 in Kinnunen-Martio [19] (or Theorem 9.12 in [6]).

\section{Perron solutions}

In addition to the assumptions given at the beginning of Sect. 3, from now on we make the convention that if $\Omega$ is unbounded, then the point at infinity, $\infty$, belongs to the boundary $\partial \Omega$. Topological notions should therefore be understood with respect to the one-point compactification $X^{*}:=X \cup\{\infty\}$.

Note that this convention does not affect any of the definitions in Sect. 2 or 3 , as $\infty$ is not added to $X$ (it is added solely to $\partial \Omega$ ).

Since continuous functions are assumed to be real-valued, every function in $C(\partial \Omega)$ is bounded even if $\Omega$ is unbounded. Note that since $X$ is second countable, so is $X^{*}$, and hence $X^{*}$ is metrizable by Urysohn's metrization theorem, see, e.g., Munkres [24, Theorems 32.3 and 34.1].

We will only consider Perron solutions and $p$-harmonic measures with respect to $\Omega$ and therefore omit $\Omega$ from the notation below.

Definition 4.1 Given a function $f: \partial \Omega \rightarrow \overline{\mathbb{R}}$, let $\mathscr{U}_{f}$ be the collection of all functions $u$ that are superharmonic in $\Omega$, bounded from below, and such that

$$
\liminf _{\Omega \ni y \rightarrow x} u(y) \geq f(x) \quad \text { for all } x \in \partial \Omega .
$$


The upper Perron solution of $f$ is defined by

$$
\bar{P} f(x)=\inf _{u \in \mathscr{U}_{f}} u(x), \quad x \in \Omega .
$$

The lower Perron solution can be defined similarly using subharmonic functions, or by letting $\underline{P} f=-\bar{P}(-f)$. If $\bar{P} f=\underline{P} f$, then we denote the common value by $P f$. Moreover, if $P f$ is real-valued, then $f$ is said to be resolutive (with respect to $\Omega$ ).

An immediate consequence of the definition is that $\bar{P} f \leq \bar{P} h$ whenever $f \leq h$ on $\partial \Omega$. Moreover, if $\alpha \in \mathbb{R}$ and $\beta \geq 0$, then $\bar{P}(\alpha+\beta f)=\alpha+\beta \bar{P} f$. Corollary 6.3 in Hansevi [16] shows that $P f \leq \bar{P} f$. In each component of $\Omega, \bar{P} f$ is either $p$-harmonic or identically $\pm \infty$, by Theorem 4.1 in Björn-Björn-Shanmugalingam [9] (or Theorem 10.10 in [6]); the proof is local and applies also to unbounded $\Omega$.

Definition 4.2 Assume that $\Omega$ is unbounded. Then $\Omega$ is p-parabolic if for every compact $K \subset \Omega$, there exist functions $u_{j} \in N^{1, p}(\Omega)$ such that $u_{j} \geq 1$ on $K$ for all $j=1,2, \ldots$, and

$$
\int_{\Omega} g_{u_{j}}^{p} d \mu \rightarrow 0 \quad \text { as } j \rightarrow \infty
$$

Otherwise, $\Omega$ is p-hyperbolic.

For examples of $p$-parabolic sets, see, e.g., Hansevi [16]. The main reason for introducing $p$-parabolic sets in [16] was to be able to obtain resolutivity results, and in particular, establishing the following resolutivity and invariance result for $p$-parabolic unbounded sets. The first such invariance result for $p$-harmonic functions was obtained, for bounded sets, by Björn-Björn-Shanmugalingam [9].

Theorem 4.3 ([9, Theorem 6.1] and [16, Theorem 7.8]) Assume that $\Omega$ is bounded or p-parabolic. Let $h: \partial \Omega \rightarrow \overline{\mathbb{R}}$ be 0 q.e. on $\partial \Omega \backslash\{\infty\}$ and $f \in C(\partial \Omega)$. Then $f$ and $f+h$ are resolutive and $P(f+h)=P f$.

Resolutivity of continuous functions is not known for unbounded $p$-hyperbolic sets, but it is rather trivial to show that constant functions are resolutive. We shall show that a similar invariance result as in Theorem 4.3 can be obtained for constant functions on unbounded $p$-hyperbolic sets. This fact will be an important tool when characterizing semiregular boundary points.

We first need to define $p$-harmonic measures, which despite the name are (usually) not measures, but nonlinear generalizations of the harmonic measure.

Definition 4.4 The upper and lower p-harmonic measures of $E \subset \partial \Omega$ are

$$
\bar{\omega}(E):=\bar{P} \chi_{E} \quad \text { and } \quad \underline{\omega}(E):=\underline{P} \chi_{E},
$$

respectively.

Proposition 4.5 Let $E \subset \partial \Omega \backslash\{\infty\}, a \in \mathbb{R}$, and $f: \partial \Omega \rightarrow \overline{\mathbb{R}}$ be such that $C_{p}(E)=0$ and $f(x)=$ a for all $x \in \partial \Omega \backslash E$. Then $P f \equiv a$. 
In particular, $\bar{\omega}(E)=\underline{\omega}(E) \equiv 0$.

Proof Without loss of generality we may assume that $a=0$. As the capacity $C_{p}$ is an outer capacity, by Corollary 1.3 in Björn-Björn-Shanmugalingam [10] (or [6, Theorem 5.31]), we can find open sets $G_{j}^{\prime} \supset E$ such that $C_{p}\left(G_{j}^{\prime}\right)<2^{-j-1}, j=1,2, \ldots$. From the decreasing sequence $\left\{\bigcup_{k=j}^{\infty} G_{k}^{\prime}\right\}_{j=1}^{\infty}$, we can choose a decreasing subsequence of open sets $G_{k}$ with $C_{p}\left(G_{k}\right)<2^{-k p}, k=1,2, \ldots$. By Lemma 5.3 in Björn-Björn-Shanmugalingam [9] (or [6, Lemma 10.17]), there is a decreasing sequence $\left\{\psi_{j}\right\}_{j=1}^{\infty}$ of nonnegative functions such that $\lim _{j \rightarrow \infty}\left\|\psi_{j}\right\|_{N^{1, p}(X)}=0$ and $\psi_{j} \geq k-j$ in $G_{k}$ whenever $k>j$. In particular, $\psi_{j}=\infty$ on $E$ for each $j=1,2, \ldots$.

Let $u_{j}$ be the lsc-regularized solution of the $\mathscr{K}_{\psi_{j}, 0}(\Omega)$-obstacle problem, $j=1,2, \ldots$ As $u_{j}$ is lsc-regularized and $u_{j} \geq \psi_{j}$ q.e., we see that $u_{j} \geq k-j$ everywhere in $G_{k}$ whenever $k>j$, and also that $u_{j} \geq 0$ everywhere in $\Omega$. In particular, $\liminf _{\Omega \ni y \rightarrow x} u_{j}(y)=\infty$ for $x \in E$, which shows that $u_{j} \in \mathscr{U}_{f}(\Omega)$ and thus $u_{j} \geq \bar{P} f$.

On the other hand, Theorem 3.2 in Hansevi [16] shows that the sequence $u_{j}$ decreases q.e. to 0 , and hence $\bar{P} f \leq 0$ q.e. in $\Omega$. Since $\bar{P} f$ is continuous, we get that $\bar{P} f \leq 0$ everywhere in $\Omega$. Applying this to $-f$ shows that $\underline{P} f=-\bar{P}(-f) \geq 0$ everywhere in $\Omega$, which together with the inequality $\underline{P} f \leq \bar{P} f$ shows that $\underline{P} f=\bar{P} f \equiv 0$. In particular, $\underline{\omega}(E)=\underline{P} \chi_{E} \equiv 0$ and $\bar{\omega}(E)=\bar{P} \chi_{E} \equiv 0$.

We will also need the following result.

Proposition 4.6 If $f: \partial \Omega \rightarrow[-\infty, \infty)$ is an upper semicontinuous function, then

$$
\bar{P} f=\inf _{C(\partial \Omega) \ni \varphi \geq f} \bar{P} \varphi \text {. }
$$

Proof Let $\mathscr{F}=\{\varphi \in C(\partial \Omega): \varphi \geq f\}$. Then $\mathscr{F}$ is downward directed, i.e., for each pair of functions $u, v \in \mathscr{F}$ there is a function $w \in \mathscr{F}$ such that $w \leq \min \{u, v\}$. Because $f$ is upper semicontinuous, $\partial \Omega$ is compact, and $X^{*}$ is metrizable, it follows from Proposition 1.12 in [6] that $f=\inf _{\varphi \in \mathscr{F}} \varphi$. Hence by Lemma 10.31 in [6] (whose proof is valid also for unbounded $\Omega) \bar{P} f=\inf _{\varphi \in \mathscr{F}} \bar{P} \varphi$.

\section{Boundary regularity}

It is not known whether continuous functions are resolutive also with respect to unbounded $p$-hyperbolic sets. We therefore define regular boundary points in the following way.

Definition 5.1 We say that a boundary point $x_{0} \in \partial \Omega$ is regular if

$$
\lim _{\Omega \ni y \rightarrow x_{0}} \bar{P} f(y)=f\left(x_{0}\right) \quad \text { for all } f \in C(\partial \Omega) .
$$

This can be paraphrased in the following way: A point $x_{0} \in \partial \Omega$ is regular if the following two conditions hold:

(I) For all $f \in C(\partial \Omega)$ the limit

$$
\lim _{\Omega \ni y \rightarrow x_{0}} \bar{P} f(y) \quad \text { exists. }
$$


(II) For all $f \in C(\partial \Omega)$ there is a sequence $\left\{y_{j}\right\}_{j=1}^{\infty}$ in $\Omega$ such that

$$
\lim _{j \rightarrow \infty} y_{j}=x_{0} \quad \text { and } \quad \lim _{j \rightarrow \infty} \bar{P} f\left(y_{j}\right)=f\left(x_{0}\right) .
$$

Furthermore, we say that a boundary point $x_{0} \in \partial \Omega$ is semiregular if (I) holds but not (II); and strongly irregular if (II) holds but not (I).

We do not require $\Omega$ to be bounded in this definition, but if it is, then it follows from Theorem 6.1 in Björn-Björn-Shanmugalingam [9] (or Theorem 10.22 in [6]) that our definition coincides with the definitions of regularity in Björn-Björn-Shanmugalingam [8, 9], and Björn-Björn [5, 6], where regularity is defined using $P f$ or $H f$. Thus we can use the boundary regularity results from these papers when considering bounded sets.

Since $\bar{P} f=-P(-f)$, the same concept of regularity is obtained if we replace the upper Perron solution by the lower Perron solution in Definition 5.1.

Boundary regularity for $p$-harmonic functions on unbounded sets in metric spaces was recently studied by Björn-Hansevi [11]. We will need some of the characterizations obtained therein. For the reader's convenience we state these results here. We will not discuss regularity of the point $\infty$ in this paper. One of the important results we will need from [11] is the Kellogg property.

Theorem 5.2 (The Kellogg property) If I is the set of irregular points in $\partial \Omega \backslash\{\infty\}$, then $C_{p}(I)=0$.

Definition 5.3 A function $u$ is a barrier (with respect to $\Omega$ ) at $x_{0} \in \partial \Omega$ if

(i) $u$ is superharmonic in $\Omega$;

(ii) $\lim _{\Omega \ni y \rightarrow x_{0}} u(y)=0$;

(iii) $\liminf _{\Omega \ni y \rightarrow x} u(y)>0$ for every $x \in \partial \Omega \backslash\left\{x_{0}\right\}$.

Superharmonic functions satisfy the strong minimum principle, i.e., if $u$ is superharmonic and attains its minimum in some component $G$ of $\Omega$, then $\left.u\right|_{G}$ is constant (see Theorem 9.13 in [6]). This implies that a barrier is always nonnegative, and furthermore, that a barrier is positive if $\partial G \backslash\left\{x_{0}\right\} \neq \varnothing$ for every component $G \subset \Omega$.

The following result is a collection of the key facts we will need from Björn-Hansevi [11, Theorems 5.2, 5.3, 6.2, and 9.1].

Theorem 5.4 Let $x_{0} \in \partial \Omega \backslash\{\infty\}$ and $\delta>0$. Also define $d_{x_{0}}: X^{*} \rightarrow[0,1]$ by

$$
d_{x_{0}}(x)= \begin{cases}\min \left\{d\left(x, x_{0}\right), 1\right\} & \text { if } x \neq \infty \\ 1 & \text { if } x=\infty\end{cases}
$$

Then the following are equivalent:

(a) The point $x_{0}$ is regular.

(b) There is a barrier at $x_{0}$.

(c) There is a positive continuous barrier at $x_{0}$.

(d) The point $x_{0}$ is regular with respect to $\Omega \cap B\left(x_{0}, \delta\right)$.

(e) It is true that 


$$
\lim _{\Omega \ni y \rightarrow x_{0}} \bar{P} f(y)=f\left(x_{0}\right)
$$

for all $f: \partial \Omega \rightarrow \mathbb{R}$ that are bounded on $\partial \Omega$ and continuous at $x_{0}$.

(f) It is true that

$$
\lim _{\Omega \ni y \rightarrow x_{0}} \bar{P} d_{x_{0}}(y)=0 .
$$

(g) The continuous solution $u$ of the $\mathscr{K}_{d_{x_{0}}, d_{x_{0}}}$-obstacle problem, satisfies

$$
\lim _{\Omega \ni y \rightarrow x_{0}} u(y)=0 .
$$

(h) If $f \in C(\bar{\Omega}) \cap D^{p}(\Omega)$, then the continuous solution $u$ of the $\mathscr{K}_{f, f^{-}}$obstacle problem, satisfies

$$
\lim _{\Omega \ni y \rightarrow x_{0}} u(y)=f\left(x_{0}\right)
$$

\section{Semiregular and strongly irregular points}

We are now ready to start our discussion of semiregular and strongly irregular boundary points. We begin by proving Theorem 1.1.

Proof of Theorem 1.1 We consider two complementary cases.

Case 1: There exists $r>0$ such that $C_{p}(B \cap \partial \Omega)=0$, where $B:=B\left(x_{0}, r\right)$.

Let $G$ be the component of $B$ containing $x_{0}$. Since $X$ is quasiconvex, by, e.g., Theorem 4.32 in [6], and thus locally connected, it follows that $G$ is open. Let $F=G \backslash \Omega$. Then

$$
C_{p}(G \cap \partial F)=C_{p}(G \cap \partial \Omega) \leq C_{p}(B \cap \partial \Omega)=0,
$$

and hence $C_{p}(F)=0$, by Lemma 8.6 in Björn-Björn-Shanmugalingam [9] (or Lemma 4.5 in [6]).

Let $f \in C(\partial \Omega)$. Then the Perron solution $\bar{P} f$ is bounded (as $f$ is bounded), and thus $\bar{P} f$ has a $p$-harmonic extension $U$ to $\Omega \cup G$, by Theorem 6.2 in Björn [3] (or Theorem 12.2 in [6]). Since $U$ is continuous, it follows that

$$
\lim _{\Omega \ni y \rightarrow x_{0}} \bar{P} f(y)=\lim _{\Omega \ni y \rightarrow x_{0}} U(y)=U\left(x_{0}\right),
$$

i.e., condition (I) in Definition 5.1 holds, and hence $x_{0}$ is either regular or semiregular.

To show that $x_{0}$ must be semiregular, we let $f(x)=\left(1-d_{x_{0}}(x) / \min \{r, 1\}\right)_{+}$on $\partial \Omega$, where $d_{x_{0}}$ is defined by (5.1). Then $f=0$ q.e. on $\partial \Omega$, and Proposition 4.5 shows that $P f \equiv 0$. Since

$$
\lim _{\Omega \ni y \rightarrow x_{0}} \bar{P} f(y)=0 \neq 1=f\left(x_{0}\right),
$$

$x_{0}$ is not regular, and hence must be semiregular.

Case 2: For all $r>0, C_{p}\left(B\left(x_{0}, r\right) \cap \partial \Omega\right)>0$.

For every $j=1,2, \ldots, C_{p}\left(B\left(x_{0}, 1 / j\right) \cap \partial \Omega\right)>0$, and by the Kellogg property (Theorem 5.2) there exists a regular boundary point $x_{j} \in B\left(x_{0}, 1 / j\right) \cap \partial \Omega$. (We do not require the $x_{j}$ to be distinct.) 
Let $f \in C(\partial \Omega)$. Because $x_{j}$ is regular, there is $y_{j} \in B\left(x_{j}, 1 / j\right) \cap \Omega$ so that $\left|\bar{P} f\left(y_{j}\right)-f\left(x_{j}\right)\right|<1 / j$. It follows that $y_{j} \rightarrow x_{0}$ and $\bar{P} f\left(y_{j}\right) \rightarrow f\left(x_{0}\right)$ as $j \rightarrow \infty$, i.e., condition (II) in Definition 5.1 holds, and hence $x_{0}$ must be either regular or strongly irregular.

As there are no strongly irregular points in case 1 , it follows that $x_{0} \in \partial \Omega \backslash\{\infty\}$ is strongly irregular if and only if $x_{0} \in \bar{R} \backslash R$, where $R:=\{x \in \partial \Omega \backslash\{\infty\}: x$ is regular $\}$ and $\bar{R}$ is the closure of $R$. And since there are no semiregular points in case 2, the set $S$ in (1.1) consists exactly of all semiregular boundary points of $\partial \Omega \backslash\{\infty\}$.

In fact, in case 2 it is possible to improve upon the result above. The sequence $\left\{y_{j}\right\}_{j=1}^{\infty}$ can be chosen independently of $f$, see the characterization (c) in Theorem 6.4.

We will characterize semiregular points by a number of equivalent conditions in Theorem 6.4. But first we obtain the following characterizations of relatively open sets of semiregular points.

Theorem 6.1 Let $V \subset \partial \Omega \backslash\{\infty\}$ be relatively open. Then the following statements are equivalent:

(a) The set $V$ consists entirely of semiregular points.

(b) The set $V$ does not contain any regular point.

(c) The capacity $C_{p}(V)=0$.

(d) The upper p-harmonic measure $\bar{\omega}(V) \equiv 0$.

(e) The lower p-harmonic measure $\underline{\omega}(V) \equiv 0$.

(f) The set $\Omega \cup V$ is open in $X, C_{p}(X \backslash(\Omega \cup V))>0, \mu(V)=0$, and every function that is bounded and superharmonic in $\Omega$ has a superharmonic extension to $\Omega \cup V$.

(g) The set $\Omega \cup V$ is open in $X, C_{p}(X \backslash(\Omega \cup V))>0$, and every function that is bounded and p-harmonic in $\Omega$ has a p-harmonic extension to $\Omega \cup V$.

If moreover $\Omega$ is bounded or p-parabolic, then also the following statements are equivalent to the statements above.

(h) For every function $f$ on $\partial \Omega$ that is continuous on $\partial \Omega \backslash V$, the Perron solution Pf depends only on $\left.f\right|_{\partial \Omega \backslash V}$ (i.e., if $f_{1}$ and $f_{2}$ are functions on $\partial \Omega$ that are continuous on $\partial \Omega \backslash V$ and such that $f_{1}=f_{2}$ on $\partial \Omega \backslash V$, then $P f_{1} \equiv P f_{2}$ ).

(i) For every $f \in C(\partial \Omega)$, the Perron solution Pf depends only on $\left.f\right|_{\partial \Omega \backslash V}$.

Note that there are examples of sets with positive capacity and even positive measure which are removable for bounded $p$-harmonic functions, see Section 9 in Björn [3] (or [6, Section 12.3]). For superharmonic functions it is not known whether such examples exist. This motivates the formulations of (f) and (g).

The following example shows that the condition $C_{p}(X \backslash(\Omega \cup V))>0$ cannot be dropped from (g), nor from (j) in Theorem 6.4 below. We do not know whether the conditions $C_{p}(X \backslash(\Omega \cup V))>0$ and $\mu(V)=0$ can be dropped from (f), but they are needed for our proof. Similarly they are needed in (1) in Theorem 6.4 below.

The condition $C_{p}(X \backslash(\Omega \cup V))>0$ was unfortunately overlooked in Björn [4] and in Björn-Björn [6]: It should be added to conditions $\left(\mathrm{d}^{\prime}\right)$ and $\left(\mathrm{e}^{\prime}\right)$ in [4, Theorem 3.1], to (h) and (i) in [4, Theorem 3.3], to $\left(\mathrm{f}^{\prime}\right)$ and $\left(\mathrm{g}^{\prime}\right)$ in [6, Theorem 13.5], and to (j) and (l) in [6, Theorem 13.10]. 
Example 6.2 Let $X=[0,1]$ be equipped with the Lebesgue measure, and let $1<p<\infty$, $\Omega=(0,1]$ and $V=\{0\}$. Then $C_{p}(V)>0$. In this case the $p$-harmonic functions on $\Omega$ are just the constant functions, and these trivially have $p$-harmonic extensions to $X$. Thus the condition $C_{p}(X \backslash(\Omega \cup V))>0$ cannot be dropped from (g).

On the other hand, the set $V$ is not removable for bounded superharmonic functions on $\Omega$, see Example 9.1 in Björn [3] or Example 12.17 in [6].

Proof of Theorem 6.1 (b) $\Rightarrow$ (c) This follows from the Kellogg property (Theorem 5.2).

(c) $\Rightarrow$ (d) This follows directly from Proposition 4.5 .

(d) $\Rightarrow$ (e) This is trivial.

(e) $\Rightarrow$ (b) Suppose that $x \in V$ is regular. Because $\chi_{V}$ is continuous at $x$, this yields a contradiction, as it follows from Theorem 5.4 that

$$
0=\lim _{\Omega \ni y \rightarrow x} \underline{\omega}(V)(y)=\lim _{\Omega \ni y \rightarrow x} \underline{P} \chi_{V}(y)=-\lim _{\Omega \ni y \rightarrow x} \bar{P}\left(-\chi_{V}\right)(y)=\chi_{V}(x)=1 .
$$

Thus $V$ does not contain any regular point.

(c) $\Rightarrow$ (f) Suppose that $C_{p}(V)=0$. Then $C_{p}(X \backslash(\Omega \cup V))=C_{p}(X \backslash \Omega)>0$ and $\mu(V)=0$. Let $x \in V$ and let $G$ be a connected neighbourhood of $x$ such that $G \cap \partial \Omega \subset V$. Sets of capacity zero cannot separate space, by Lemma 4.6 in Björn-Björn [6], and hence $G \backslash \partial \Omega$ must be connected, i.e., $G \subset \bar{\Omega}$, from which it follows that $\Omega \cup V$ is open in $X$. The superharmonic extension is now provided by Theorem 6.3 in Björn [3] (or Theorem 12.3 in [6]).

(f) $\Rightarrow$ (g) Let $u$ be a bounded $p$-harmonic function on $\Omega$. Then, by assumption, $u$ has a superharmonic extension $U$ to $\Omega \cup V$. Moreover, as $-u$ is also bounded and $p$-harmonic, there is a superharmonic extension $W$ of $-u$ to $\Omega \cup V$. Now, as $-W$ is clearly a subharmonic extension of $u$ to $\Omega \cup V$, Proposition 6.5 in Björn [3] (or Proposition 12.5 in [6]) asserts that $U=-W$ is $p$-harmonic (it is here that we use that $\mu(V)=0$ ).

(g) $\Rightarrow$ (a) Let $x_{0} \in V$. Since $\Omega \cup V$ is open in $X$, we see that $V \cap \partial(\Omega \cup V)=\varnothing$, and hence $x_{0} \notin \partial(\Omega \cup V)$. Let

$$
h(x)=\left(1-\frac{d_{x_{0}}(x)}{\operatorname{dist}_{x_{0}}(\partial(\Omega \cup V))}\right)_{+}, \quad x \in \partial \Omega,
$$

where $d_{x_{0}}$ is defined by (5.1), and $\operatorname{dist}_{x_{0}}(E):=\inf _{y \in E} d_{x_{0}}(y), E \subset X^{*}$. Then $\bar{P} h$ is bounded and has a $p$-harmonic extension $U$ to $\Omega \cup V$, and hence the Kellogg property (Theorem 5.2) implies that

$$
\lim _{\Omega \cup V \ni y \rightarrow x} U(y)=\lim _{\Omega \ni y \rightarrow x} \bar{P} h(y)=h(x)=0 \quad \text { for q.e. } x \in \partial(\Omega \cup V) \backslash\{\infty\} .
$$

Let $G$ be the component of $\Omega \cup V$ containing $x_{0}$. Then

$$
C_{p}(X \backslash G) \geq C_{p}(X \backslash(\Omega \cup V))>0 .
$$

It then follows from Lemma 4.3 in Björn-Björn [5] (or Lemma 4.5 in [6]) that $C_{p}(\partial G)>0$. In particular, it follows from (6.1) that $U \not \equiv 1$ in $G$, and thus, by the strong maximum principle (see Corollary 6.4 in Kinnunen-Shanmugalingam [20] or [6, Theorem 8.13]), that $U\left(x_{0}\right)<1$. Therefore

$$
\lim _{\Omega \ni y \rightarrow x_{0}} \bar{P} h(y)=U\left(x_{0}\right)<1=h\left(x_{0}\right)
$$


and hence $x_{0}$ must be irregular.

However, if $f \in C(\partial \Omega)$, then $\bar{P} f$ has a $p$-harmonic extension $W$ to $\Omega \cup V$. Since $W$ is continuous in $\Omega \cup V$, it follows that

$$
\lim _{\Omega \ni y \rightarrow x_{0}} \bar{P} f(y)=W\left(x_{0}\right),
$$

and hence the limit on the left-hand side always exists. Thus $x_{0}$ is semiregular.

(a) $\Rightarrow$ (b) This is trivial.

We now assume that $\Omega$ is bounded or $p$-parabolic.

(c) $\Rightarrow$ (h) Suppose that the functions $f_{1}$ and $f_{2}$ on $\partial \Omega$ are continuous on $\partial \Omega \backslash V$ and that $f_{1}=f_{2}$ on $\partial \Omega \backslash V$. Since $V$ is relatively open in $\partial \Omega \backslash\{\infty\}$, by Urysohn's lemma, see, e.g., Munkres [24, Theorem 33.1], there exists $f \in C(\partial \Omega)$ such that $f=f_{1}=f_{2}$ on $\partial \Omega \backslash V$. Let $h_{1}=f_{1}-f$ and $h_{2}=f_{2}-f$. Then $h_{1}$ and $h_{2}$ are equal to 0 q.e. on $\partial \Omega$, as $C_{p}(V)=0$. By Theorem 4.3, it follows that $P f_{1}=P\left(f+h_{1}\right)=P f=P\left(f+h_{2}\right)=P f_{2}$.

(h) $\Rightarrow$ (i) This is trivial.

(i) $\Rightarrow$ (e) As $-\chi_{V}: \partial \Omega \rightarrow \mathbb{R}$ is upper semicontinuous, it follows from Proposition 4.6, and (h), that

$$
0 \leq \underline{\omega}(V)=-\bar{P}\left(-\chi_{V}\right)=-\inf _{\substack{\varphi \in C(\partial \Omega) \\-\chi_{V} \leq \varphi \leq 0}} \bar{P} \varphi=0,
$$

and hence $\underline{\omega}(V)=0$.

Definition 6.3 A function $u$ is a semibarrier (with respect to $\Omega$ ) at $x_{0} \in \partial \Omega$ if

(i) $\quad u$ is superharmonic in $\Omega$;

(ii) $\liminf _{\Omega \ni y \rightarrow x_{0}} u(y)=0$;

(iii) $\liminf _{\Omega \ni y \rightarrow x} u(y)>0$ for every $x \in \partial \Omega \backslash\left\{x_{0}\right\}$.

Moreover, we say that $u$ is a weak semibarrier (with respect to $\Omega$ ) at $x_{0} \in \partial \Omega$ if $u$ is a positive superharmonic function such that (ii) holds.

Now we are ready to characterize the semiregular points by means of capacity, $p$-harmonic measures, removable singularities, and semibarriers. In particular, we show that semiregularity is a local property.

Theorem 6.4 Let $x_{0} \in \partial \Omega \backslash\{\infty\}, \delta>0$, and $d_{x_{0}}: X^{*} \rightarrow[0,1]$ be defined by (5.1). Then the following statements are equivalent:

(a) The point $x_{0}$ is semiregular.

(b) The point $x_{0}$ is semiregular with respect to $G:=\Omega \cap B\left(x_{0}, \delta\right)$.

(c) There is no sequence $\left\{y_{j}\right\}_{j=1}^{\infty}$ in $\Omega$ such that $y_{j} \rightarrow x_{0}$ as $j \rightarrow \infty$ and

$$
\lim _{j \rightarrow \infty} \bar{P} f\left(y_{j}\right)=f\left(x_{0}\right) \quad \text { for all } f \in C(\partial \Omega) .
$$

(d) The point $x_{0}$ is neither regular nor strongly irregular.

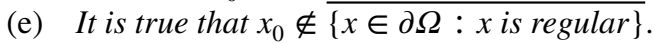

(f) There is a neighbourhood $V$ of $x_{0}$ such that $C_{p}(V \cap \partial \Omega)=0$.

(g) There is a neighbourhood $V$ of $x_{0}$ such that $C_{p}(V \backslash \Omega)=0$. 
(h) There is a neighbourhood $V$ of $x_{0}$ such that $\bar{\omega}(V \cap \partial \Omega) \equiv 0$.

(i) There is a neighbourhood $V$ of $x_{0}$ such that $\underline{\omega}(V \cap \partial \Omega) \equiv 0$.

(j) There is a neighbourhood $V \subset \bar{\Omega}$ of $x_{0}$, with $C_{p}(X \backslash(\Omega \cup V))>0$, such that every function that is bounded and p-harmonic in $\Omega$ has a p-harmonic extension to $\Omega \cup V$.

(k) There is a neighbourhood $V$ of $x_{0}$ such that every function that is bounded and p-harmonic in $\Omega$ has a p-harmonic extension to $\Omega \cup V$, and moreover $x_{0}$ is irregular.

(1) There is a neighbourhood $V$ of $x_{0}$, with $C_{p}(X \backslash(\Omega \cup V))>0$ and $\mu(V \backslash \Omega)=0$, such that every function that is bounded and superharmonic in $\Omega$ has a superharmonic extension to $\Omega \cup V$.

(m) It is true that

$$
\lim _{\Omega \ni y \rightarrow x_{0}} \bar{P} d_{x_{0}}(y)>0
$$

(n) It is true that

$$
\liminf _{\Omega \ni y \rightarrow x_{0}} \bar{P} d_{x_{0}}(y)>0 .
$$

(o) There is no weak semibarrier at $x_{0}$.

(p) There is no semibarrier at $x_{0}$.

(q) The continuous solution of the $\mathscr{K}_{d_{x_{0}}, d_{x_{0}}}$-obstacle problem is not a semibarrier at $x_{0}$.

If moreover $\Omega$ is bounded or p-parabolic, then also the following statements are equivalent to the statements above.

(r) There is a neighbourhood $V$ of $x_{0}$ such that for every function $f$ on $\partial \Omega$ that is continuous on $\partial \Omega \backslash V$, the Perron solution Pf depends only on $\left.f\right|_{\partial \Omega \backslash V}$ (i.e., if $f_{1}$ and $f_{2}$ are functions on $\partial \Omega$ that are continuous on $\partial \Omega \backslash V$ and such that $f_{1}=f_{2}$ on $\partial \Omega \backslash V$, then $\left.P f_{1} \equiv P f_{2}\right)$.

(s) There is a neighbourhood $V$ of $x_{0}$ such that for every $f \in C(\partial \Omega)$, the Perron solution Pf depends only on $\left.f\right|_{\partial \Omega \backslash V}$.

Proof (e) $\Leftrightarrow$ (f) $\Leftrightarrow$ (h) $\Leftrightarrow$ (i) $\Rightarrow$ (a) This follows directly from Theorem 6.1, with $V$ therein corresponding to $V \cap \partial \Omega$ here.

(a) $\Rightarrow(\mathrm{m})$ Since $x_{0}$ is semiregular, the limit

$$
\alpha:=\lim _{\Omega \ni y \rightarrow x_{0}} \bar{P} d_{x_{0}}(y)
$$

exists. If $\alpha=0$, then $x_{0}$ must be regular by Theorem 5.4 , which is a contradiction. Hence $\alpha>0$.

$(\mathrm{m}) \Rightarrow(\mathrm{n}) \Rightarrow(\mathrm{d}) \Rightarrow(\mathrm{c})$ These implications are trivial.

$\neg(\mathrm{e}) \Rightarrow \neg(\mathrm{c})$ Suppose that $x_{0} \in \overline{\{x \in \partial \Omega: x \text { is regular }\}}$. For each integer $j \geq 2$, there exists a regular point $x_{j} \in B\left(x_{0}, 1 / j\right) \cap \partial \Omega$. Define $f_{j} \in C(\partial \Omega)$ by letting

$$
f_{j}(x)=\left(j d_{x_{0}}(x)-1\right)_{+}, \quad j=2,3, \ldots
$$

Because $x_{j}$ is regular, there is $y_{j} \in B\left(x_{j}, 1 / j\right) \cap \Omega$ such that

$$
\left|\bar{P} f_{j}\left(y_{j}\right)\right|=\left|f_{j}\left(x_{j}\right)-\bar{P} f_{j}\left(y_{j}\right)\right|<1 / j .
$$

Hence $y_{j} \rightarrow x_{0}$ and $\bar{P} f_{j}\left(y_{j}\right) \rightarrow 0$ as $j \rightarrow \infty$. 
Let $f \in C(\partial \Omega)$ and $\alpha:=f\left(x_{0}\right)$. Let $\varepsilon>0$. Then we can find an integer $k \geq 2$ such that $|f-\alpha| \leq \varepsilon$ on $B\left(x_{0}, 2 / k\right) \cap \partial \Omega$. Choose $m$ such that $|f-\alpha| \leq m$. It follows that $f-\alpha \leq m f_{j}+\varepsilon$ for every $j \geq k$, and thus

$$
\limsup _{j \rightarrow \infty} \bar{P} f\left(y_{j}\right) \leq \limsup _{j \rightarrow \infty} \bar{P}\left(m f_{j}+\alpha+\varepsilon\right)\left(y_{j}\right)=m \lim _{j \rightarrow \infty} \bar{P} f_{j}\left(y_{j}\right)+\alpha+\varepsilon=\alpha+\varepsilon .
$$

Letting $\varepsilon \rightarrow 0$ shows that $\lim \sup _{j \rightarrow \infty} \bar{P} f\left(y_{j}\right) \leq \underline{\alpha}$.

Applying this to $\tilde{f}=-f$ yields $\lim \sup _{j \rightarrow \infty} \bar{P} \tilde{f}\left(y_{j}\right) \leq-\alpha$. It follows that

$$
\liminf _{j \rightarrow \infty} \bar{P} f\left(y_{j}\right) \geq \liminf _{j \rightarrow \infty} \underline{P} f\left(y_{j}\right)=-\limsup _{j \rightarrow \infty} \bar{P} \tilde{f}\left(y_{j}\right) \geq \alpha,
$$

and hence $\lim _{j \rightarrow \infty} \bar{P} f\left(y_{j}\right)=f\left(x_{0}\right)$.

(f) $\Leftrightarrow$ (b) Observe that (f) is equivalent to the existence of a neighbourhood $U$ of $x_{0}$ with $C_{p}(U \cap \partial G)=0$, which is equivalent to (b), by the already proved equivalence (f) $\Leftrightarrow$ (a) applied to $G$ instead of $\Omega$.

(f) $\Rightarrow(\mathrm{g})$ Let $V$ be a neighbourhood of $x_{0}$ such that $C_{p}(V \cap \partial \Omega)=0$. By Theorem 6.1, (c) $\Rightarrow(\mathrm{f})$, the set $U:=\Omega \cup(V \cap \partial \Omega)$ is open and $C_{p}(U \backslash \Omega)=0$.

(g) $\Rightarrow$ (f) This is trivial.

(g) $\Leftrightarrow(\mathrm{j}) \Leftrightarrow(\mathrm{l})$ In all three statements it follows directly that $V \subset \bar{\Omega}$. Thus their equivalence follows directly from Theorem 6.1, with $V$ in Theorem 6.1 corresponding to $V \cap \partial \Omega$ here.

$(\mathrm{j}) \Rightarrow(\mathrm{k})$ We only have to show the last part, i.e., that $x_{0}$ is irregular, but this follows from the already proved implication $(\mathrm{j}) \Rightarrow(\mathrm{a})$.

(k) $\Rightarrow$ (a) Let $f \in C(\partial \Omega)$. Then $\bar{P} f$ has a $p$-harmonic extension $U$ to $\Omega \cup V$ for some neighbourhood $V$ of $x_{0}$, and hence

$$
\lim _{\Omega \ni y \rightarrow x_{0}} \bar{P} f(y)=U\left(x_{0}\right) .
$$

Since $x_{0}$ is irregular it follows that $x_{0}$ must be semiregular.

(1) $\Rightarrow$ (o) Let $u$ be a positive superharmonic function on $\Omega$. Then $\min \{u, 1\}$ is superharmonic by Lemma 9.3 in Björn-Björn [6], and hence has a superharmonic extension $U$ to $\Omega \cup V$. As $U$ is lsc-regularized (see Sect. 3) and $\mu(V \backslash \Omega)=0$, it follows that $U \geq 0$ in $\Omega \cup V$. Suppose that $U\left(x_{0}\right)=0$. Then the strong minimum principle [6, Theorem 9.13] implies that $U \equiv 0$ in the component of $\Omega \cup V$ that contains $x_{0}$. But this is in contradiction with $u$ being positive in $\Omega$, and thus

$$
\liminf _{\Omega \ni y \rightarrow x_{0}} u(y) \geq U\left(x_{0}\right)>0 .
$$

Thus there is no weak semibarrier at $x_{0}$.

$\neg(\mathrm{p}) \Rightarrow \neg(\mathrm{o})$ Let $u$ be a semibarrier at $x_{0}$. If $u>0$ in all of $\Omega$, then $u$ is a weak semibarrier at $x_{0}$. On the other hand, assume that there exists $x \in \Omega$ such that $u(x)=0$ (in this case $u$ is not a weak semibarrier). Then the strong minimum principle [6, Theorem 9.13] implies that $u \equiv 0$ in the component $G \subset \Omega$ that contains $x$, and hence $x_{0}$ must be the only boundary point of $G$, because $u$ is a semibarrier. As $C_{p}(X \backslash G) \geq C_{p}(X \backslash \Omega)>0$, Lemma 4.3 in Björn-Björn [5] (or Lemma 4.5 in [6]) implies that $C_{p}\left(\left\{x_{0}\right\}\right)=C_{p}(\partial G)>0$. By the Kellogg property (Theorem 5.2), $x_{0}$ is regular, and hence Theorem 5.4 asserts that there is a positive barrier $v$ at $x_{0}$, and thus $v$ is a weak semibarrier.

(p) $\Rightarrow$ (q) This is trivial.

$\neg(\mathrm{e}) \Rightarrow \neg$ (q) Let $u$ be the continuous solution of the $\mathscr{K}_{d_{x_{0}}, d_{x_{0}}}$-obstacle problem, which is superharmonic (see Sect. 3). Moreover, it is clear that 


$$
\liminf _{\Omega \ni y \rightarrow x} u(y)>0 \quad \text { whenever } x \in \partial \Omega \backslash\left\{x_{0}\right\},
$$

and thus $u$ satisfies (i) and (iii) in Definition 6.3.

Let $\left\{x_{j}\right\}_{j=1}^{\infty}$ be a sequence of regular boundary points such that $d_{x_{0}}\left(x_{j}\right)<1 / j$. By Theorem 5.4, $\lim _{\Omega \ni y \rightarrow x_{j}} u(y)=d_{x_{0}}\left(x_{j}\right)$. Thus we can find $y_{j} \in B\left(x_{j}, 1 / j\right) \cap \Omega$ so that $u\left(y_{j}\right)<2 / j$. Hence $u$ satisfies (ii) in Definition 6.3 as

$$
0 \leq \liminf _{\Omega \ni y \rightarrow x_{0}} u(y) \leq \liminf _{j \rightarrow \infty} u\left(y_{j}\right)=0 .
$$

We now assume that $\Omega$ is bounded or $p$-parabolic.

(e) $\Leftrightarrow(\mathrm{r}) \Leftrightarrow$ (s) This follows directly from Theorem 6.1, with $V$ therein corresponding to $V \cap \partial \Omega$ here.

We conclude our description of boundary points with some characterizations of strongly irregular points. As for regular and semiregular points, strong irregularity is a local property.

Theorem 6.5 Let $x_{0} \in \partial \Omega \backslash\{\infty\}, \delta>0$, and $d_{x_{0}}: X^{*} \rightarrow[0,1]$ be defined by (5.1). Then the following are equivalent:

(a) The point $x_{0}$ is strongly irregular.

(b) The point $x_{0}$ is strongly irregular with respect to $G:=\Omega \cap B\left(x_{0}, \delta\right)$.

(c) The point $x_{0}$ is irregular and there exists a sequence $\left\{y_{j}\right\}_{j=1}^{\infty}$ in $\Omega$ such that $y_{j} \rightarrow x_{0}$ as $j \rightarrow \infty$, and

$$
\lim _{j \rightarrow \infty} \bar{P} f\left(y_{j}\right)=f\left(x_{0}\right) \quad \text { for all } f \in C(\partial \Omega) .
$$

(d) It is true that $x_{0} \in \bar{R} \backslash R$, where $R:=\{x \in \partial \Omega: x$ is regular $\}$.

(e) It is true that

$$
\liminf _{\Omega \ni y \rightarrow x_{0}} \bar{P} d_{x_{0}}(y)=0<\limsup _{\Omega \ni y \rightarrow x_{0}} \bar{P} d_{x_{0}}(y)
$$

(f) There exists $f \in C(\partial \Omega)$ such that

$$
\lim _{\Omega \ni y \rightarrow x_{0}} \bar{P} f(y)
$$

does not exist.

(g) The continuous solution $u$ of the $\mathscr{K}_{d_{x_{0}}, d_{x_{0}}}$-obstacle problem satisfies

$$
\liminf _{\Omega \ni y \rightarrow x_{0}} u(y)=0<\limsup _{\Omega \ni y \rightarrow x_{0}} u(y) .
$$

(h) There is a semibarrier (or equivalently there is a weak semibarrier) but no barrier at $x_{0}$.

The trichotomy property (Theorem 1.1) shows that a boundary point is either regular, semiregular, or strongly irregular. We will use this in the following proof. 
Proof (a) $\Leftrightarrow$ (b) By Theorems 5.4 and 6.4, regularity and semiregularity are local properties, and hence this must be true also for strong irregularity.

(a) $\Leftrightarrow$ (c) $\Leftrightarrow$ (d) This follows from Theorem 6.4 (a) $\Leftrightarrow$ (c) $\Leftrightarrow$ (e).

(a) $\Rightarrow$ (e) Since $x_{0}$ is strongly irregular and $\bar{P} d_{x_{0}}$ is nonnegative, it follows that

$$
\liminf _{\Omega \ni y \rightarrow x_{0}} \bar{P} d_{x_{0}}(y)=0 \leq \limsup _{\Omega \ni y \rightarrow x_{0}} \bar{P} d_{x_{0}}(y) .
$$

If lim $\sup _{\Omega \ni y \rightarrow x_{0}} \bar{P} d_{x_{0}}(y)=0$, then $x_{0}$ must be regular by Theorem 5.4, which is a contradiction. Thus

$$
\limsup _{\Omega \ni y \rightarrow x_{0}} \bar{P} d_{x_{0}}(y)>0
$$

(e) $\Rightarrow$ (f) This is trivial.

(f) $\Rightarrow$ (a) By definition, $x_{0}$ is neither regular nor semiregular, and hence must be strongly irregular.

(a) $\Leftrightarrow(\mathrm{g})$ Theorem 5.4 shows that $x_{0}$ is regular if and only if $\lim _{\Omega \ni y \rightarrow x_{0}} u(y)=0$. On the other hand, Theorem 6.4 implies that $x_{0}$ is semiregular if and only if $\liminf _{\Omega \ni y \rightarrow x_{0}} u(y)>0$. The equivalence follows by combining these two facts.

(a) $\Leftrightarrow(\mathrm{h})$ By Theorem 6.4, $x_{0}$ is semiregular if and only if there is no (weak) semibarrier at $x_{0}$. On the other hand, by Theorem 5.4, there is a barrier at $x_{0}$ if and only if $x_{0}$ is regular. Combining these two facts gives the equivalence.

Acknowledgements The first author was supported by the Swedish Research Council, Grant 2016-03424.

Funding Open access funding provided by Linköping University.

Open Access This article is licensed under a Creative Commons Attribution 4.0 International License, which permits use, sharing, adaptation, distribution and reproduction in any medium or format, as long as you give appropriate credit to the original author(s) and the source, provide a link to the Creative Commons licence, and indicate if changes were made. The images or other third party material in this article are included in the article's Creative Commons licence, unless indicated otherwise in a credit line to the material. If material is not included in the article's Creative Commons licence and your intended use is not permitted by statutory regulation or exceeds the permitted use, you will need to obtain permission directly from the copyright holder. To view a copy of this licence, visit http://creativecommons.org/licenses/by/4.0/.

\section{References}

1. Adamowicz, T., Björn, A., Björn, J.: Regularity of $p(\cdot)$-superharmonic functions, the Kellogg property and semiregular boundary points. Ann. Inst. H. Poincaré Anal. Non Linéaire 31, 1131-1153 (2014)

2. Björn, A.: Characterizations of $p$-superharmonic functions on metric spaces. Stud. Math. 169, 45-62 (2005)

3. Björn, A.: Removable singularities for bounded $p$-harmonic and quasi(super)harmonic functions on metric spaces. Ann. Acad. Sci. Fenn. Math. 31, 71-95 (2006)

4. Björn, A.: A regularity classification of boundary points for $p$-harmonic functions and quasiminimizers. J. Math. Anal. Appl. 338, 39-47 (2008)

5. Björn, A., Björn, J.: Boundary regularity for $p$-harmonic functions and solutions of the obstacle problem on metric spaces. J. Math. Soc. Jpn. 58, 1211-1232 (2006)

6. Björn, A., Björn, J.: Nonlinear Potential Theory on Metric Spaces. EMS Tracts in Mathematics, vol. 17. European Mathematical Society, Zürich (2011)

7. Björn, A., Björn, J., Li, X.: Sphericalization and p-harmonic functions on unbounded domains in Ahlfors regular metric spaces. J. Math. Anal. Appl. 474, 852-875 (2019) 
8. Björn, A., Björn, J., Shanmugalingam, N.: The Dirichlet problem for $p$-harmonic functions on metric spaces. J. Reine Angew. Math. 556, 173-203 (2003)

9. Björn, A., Björn, J., Shanmugalingam, N.: The Perron method for $p$-harmonic functions in metric spaces. J. Differ. Equ. 195, 398-429 (2003)

10. Björn, A., Björn, J., Shanmugalingam, N.: Quasicontinuity of Newton-Sobolev functions and density of Lipschitz functions on metric spaces. Houst. J. Math. 34, 1197-1211 (2008)

11. Björn, A., Hansevi, D.: Boundary regularity for $p$-harmonic functions and solutions of obstacle problems on unbounded sets in metric spaces. Anal. Geom. Metr. Spaces 7, 179-196 (2019)

12. Björn, A., Marola, N.: Moser iteration for (quasi)minimizers on metric spaces. Manuscr. Math. 121, 339-366 (2006)

13. Björn, J.: Boundary continuity for quasiminimizers on metric spaces. Ill. J. Math. 46, 383-403 (2002)

14. Björn, J., MacManus, P., Shanmugalingam, N.: Fat sets and pointwise boundary estimates for $p$-harmonic functions in metric spaces. J. Anal. Math. 85, 339-369 (2001)

15. Hansevi, D.: The obstacle and Dirichlet problems associated with $p$-harmonic functions in unbounded sets in $\mathbb{R}^{n}$ and metric spaces. Ann. Acad. Sci. Fenn. Math. 40, 89-108 (2015)

16. Hansevi, D.: The Perron method for $p$-harmonic functions in unbounded sets in $\mathbb{R}^{n}$ and metric spaces. Math. Z. 288, 55-74 (2018)

17. Heinonen, J., Koskela, P.: Quasiconformal maps in metric spaces with controlled geometry. Acta Math. 181, 1-61 (1998)

18. Heinonen, J., Koskela, P., Shanmugalingam, N., Tyson, J.T.: Sobolev Spaces on Metric Measure Spaces. New Mathematical Monographs, vol. 27. Cambridge University Press, Cambridge (2015)

19. Kinnunen, J., Martio, O.: Nonlinear potential theory on metric spaces. Ill. J. Math. 46, 857-883 (2002)

20. Kinnunen, J., Shanmugalingam, N.: Regularity of quasi-minimizers on metric spaces. Manuscr. Math. 105, 401-423 (2001)

21. Koskela, P., MacManus, P.: Quasiconformal mappings and Sobolev spaces. Stud. Math. 131, 1-17 (1998)

22. Lebesgue, H.: Conditions de régularité, conditions d'irrégularité, conditions d'impossibilité dans le problème de Dirichlet. C. R. Acad. Sci. Paris 178, 349-354 (1924)

23. Lukeš, J., Malý, J.: On the boundary behaviour of the Perron generalized solution. Math. Ann. 257, 355-366 (1981)

24. Munkres, J.R.: Topology, 2nd edn. Prentice Hall, Upper Saddle River (2000)

25. Perron, O.: Eine neue Behandlung der ersten Randwertaufgabe für $\Delta u=0$. Math. Z. 18, 42-54 (1923)

26. Remak, R.: Über potentialkonvexe Funktionen. Math. Z. 20, 126-130 (1924)

27. Shanmugalingam, N.: Newtonian spaces: an extension of Sobolev spaces to metric measure spaces. Rev. Mat. Iberoam. 16, 243-279 (2000)

28. Wiener, N.: The Dirichlet problem. J. Math. Phys. 3, 127-146 (1924)

Publisher's Note Springer Nature remains neutral with regard to jurisdictional claims in published maps and institutional affiliations. 